\title{
Prognostic Impact of Fusobacterium nucleatum Depends on Combined Tumor Location and Microsatellite Instability Status in Stage II/III Colorectal Cancers Treated with Adjuvant Chemotherapy
}

Hyeon Jeong $\mathrm{Oh}^{1} \cdot$ Jung Ho $\mathrm{Kim}^{1}$ Jeong Mo Bae ${ }^{1} \cdot$ Hyun Jung Kim ${ }^{2}$ Nam-Yun $\mathrm{Cho}^{2}$ Gyeong Hoon Kang ${ }^{1,2}$

'Department of Pathology, Seoul National University Hospital, Seoul National University College of Medicine, Seoul; ' 2 Laboratory of Epigenetics, Cancer Research Institute, Seoul National University College of Medicine, Seoul, Korea

Received: July 20, 2018

Revised: November 9, 2018

Accepted: November 29, 2018

Corresponding Author

Jung Ho Kim, MD, PhD

Department of Pathology, Seoul National University

Hospital, 101 Daehak-ro, Jongno-gu,

Seoul 03080, Korea

Tel: +82-2-2072-2828

Fax: $+82-2-743-5530$

E-mail: junghokim@snuh.org

Gyeong Hoon Kang, MD, PhD

Department of Pathology, Seoul National University

College of Medicine, 103 Daehak-ro, Jongno-gu,

Seoul 03080, Korea

Tel: +82-2-740-8263

Fax: +82-2-765-5600

E-mail: ghkang@snu.ac.kr
Background: This study aimed to investigate the prognostic impact of intratumoral Fusobacterium nucleatum in colorectal cancer (CRC) treated with adjuvant chemotherapy. Methods: $F$. nucleatum DNA was quantitatively measured in a total of 593 CRC tissues retrospectively collected from surgically resected specimens of stage III or high-risk stage II CRC patients who had received curative surgery and subsequent oxaliplatin-based adjuvant chemotherapy (either FOLFOX or CAPOX). Each case was classified into one of the three categories: F. nucleatum-high, low, or -negative. Results: No significant differences in survival were observed between the $F$. nucleatum-high and -low/negative groups in the 593 CRCs $(p=.671)$. Subgroup analyses according to tumor location demonstrated that disease-free survival was significantly better in $F$. nucleatum-high than in -low/negative patients with non-sigmoid colon cancer (including cecal, ascending, transverse, and descending colon cancers; $n=219$; log-rank $p=.026$ ). In multivariate analysis, $F$. nucleatum was determined to be an independent prognostic factor in non-sigmoid colon cancers (hazard ratio, $0.42 ; 95 \%$ confidence interval, 0.18 to $0.97 ; p=.043$ ). Furthermore, the favorable prognostic effect of $F$. nucleatum-high was observed only in a non-microsatellite instability-high (non-MSI-high) subset of non-sigmoid colon cancers (log-rank $p=0.014$ ), but not in a MSI-high subset (log-rank $p=0.844$ ), suggesting that the combined status of tumor location and MSI may be a critical factor for different prognostic impacts of $F$. nucleatum in CRCs treated with adjuvant chemotherapy. Conclusions: Intratumoral $F$. nucleatum load is a potential prognostic factor in a non-MSI-high/non-sigmoid/non-rectal cancer subset of stage II/III CRCs treated with oxaliplatin-based adjuvant chemotherapy.

Key Words: Colorectal neoplasms; Fusobacterium; Gastrointestinal microbiome; Prognosis
Accumulating evidence has implicated the gut microbiota as having various roles in carcinogenesis, prognosis, and treatment response of colorectal cancer (CRC). ${ }^{1-5}$ Among the gut microbiota, Fusobacterium nucleatum has been identified as a specifically enriched species within the tumor tissue of human CRC., ${ }^{6,7} \mathrm{Al}-$ though $F$. nucleatum composes a relatively small proportion of the normal intestinal flora, the amount of tumor-invasive $F$. nucleatum was reported to be remarkably increased in a subset of CRC cases. $^{6-9}$

In the colorectal carcinogenesis pathway, the amount of invasive F. nucleatum gradually increases from premalignant adenoma- tous lesions to carcinomas in the large intestine. ${ }^{9-11}$ Among the premalignant colorectal lesions, sessile serrated adenomas have been suggested to be closely correlated with $F$. nucleatum enrichment. ${ }^{9,10}$ Therefore, it has been suspected that $F$. nucleatum might have roles in the serrated carcinogenesis pathway of the colorectum. However, detailed mechanisms of the increase of invasive $F$. nucleatum abundance and pathobiological implications of $F$. nucleatum in the serrated pathway are unclear. Experimental data indicate that $F$. nucleatum might have carcinogenic roles through the modulation of the $\mathrm{E}$-cadherin/ $\beta$-catenin signalling pathway and/or promotion of the pro-inflammatory micro- 
environment. ${ }^{1,2}$ However, these biological mechanisms cannot fully explain the basis of the association of $F$. nucleatum with the serrated pathway in CRC.

The findings using clinical samples support the suggestion that a high load of intratumoral $F$. nucleatum is associated with various clinicopathological and molecular features of CRC, including right-sided tumor location, poor prognosis, poor response to chemotherapy, low density of $\mathrm{CD}^{+}$tumor-infiltrating lymphocytes, high density of tumor-infiltrating macrophages, $\mathrm{CpG}$ island methylator phenotype (CIMP), and microsatellite instability (MSI) ${ }^{3,4,8,12-14}$ However, these observed associations of $F$. nucleatum in CRC are less robust, since the results were derived from limited study cohorts. Thus, precise clinicopathological and molecular implications of $F$. nucleatum-high CRC need to be elucidated and validated using additional independent data.

Recent studies reported that the gut microbiota is associated with responses to chemotherapy and immunotherapy in solid tumors. ${ }^{4,5,15-17}$ Especially, Yu et al. ${ }^{4}$ reported that $F$. nucleatum can promote chemoresistance in CRC by modulating the Toll-like receptor, micro-RNAs, and autophagy pathways. Based on these results, we designed a study to investigate the prognostic impacts of $F$. nucleatum in CRC patients treated with adjuvant chemotherapy. The amount of intratumoral $F$. nucleatum and its prognostic associations were analyzed in a total of 593 stage III or high-risk stage II CRCs treated with adjuvant FOLFOX (folinic acid/5-fluorouracil plus oxaliplatin) or CAPOX (capecitabine plus oxaliplatin) chemotherapy.

\section{MATERIALS AND METHODS}

\section{Case selection}

Formalin-fixed, paraffin-embedded (FFPE) tissues of 747 consecutive series of primary CRCs were collected from the pathology archive of Seoul National University Hospital, Seoul, Korea. All the tissues were from surgical specimens of patients who underwent curative surgery and subsequent adjuvant chemotherapy for stage III or high-risk stage II CRC at Seoul National University Hospital from 2005 to 2012. The inclusion criteria for the case selection were age greater than 18 years, adenocarcinoma histology without neuroendocrine or squamous cell component, stage III or high-risk stage II according to pathological staging, complete resection (R0) of the primary tumor with tumorfree resection margins, and the completion of at least six cycles of adjuvant FOLFOX chemotherapy or four cycles of adjuvant CAPOX therapy. The criteria for high-risk stage II were tumor invasion into visceral peritoneum or direct invasion into adjacent organs/structures (pT4), clinically obstruction or perforation, poorly differentiated or undifferentiated histology (G3/G4), lymphovascular invasion, and perineural invasion. The patients who received pre-operative neoadjuvant chemotherapy and/or radiotherapy (especially patients with rectal cancer) and patients with a history of other malignancy within 5 years were excluded. Initially, 747 cases were subjected to quantitative polymerase chain reaction (qPCR) analysis for $F$. nucleatum. Among them, 154 inadequate samples determined by invalid or poor quality results from the qPCR analysis, as described subsequently, were excluded. Finally, a total of 593 CRC cases were analyzed. The Institutional Review Board of our hospital approved this study (IRB No. 1805-018-944). The Institutional Review Board exempted our study from obtaining informed consent from patients because our study was a retrospective, anonymous, tissuebased investigation.

\section{Clinicopathological data}

Clinical data, including age, sex, tumor location, and gross tumor type, were collected from electronic medical records. Hematoxylin and eosin-stained tissue slides of each case were independently reviewed by pathologists (J.M.B. and G.H.K.) to evaluate histopathological features, including $\mathrm{pT} / \mathrm{pN}$ categories, tumor grade, lymphovascular invasion, perineural invasion, and mucinous histology.

\section{qPCR for $F$. nucleatum}

Genomic DNA extraction from FFPE tissues of the 747 CRCs and qPCR for $F$. nucleatum, using the 747 tumor DNA samples, were conducted as previously described. ${ }^{14}$ In brief, the following primers and probes targeting the 16S rRNA gene DNA sequence of $F$. nucleatum and the reference gene (prostaglandin transporter, PGT), were used: F. nucleatum forward primer, 5'-CAACCATTACTTTAACTCTACCATGTTCA-3'; F. nucleatum reverse' primer, 5'-GTTGACTTTACAGAAGGAGATTATGTAAAAATC-3'; F. nucleatum FAM probe, 5'-GTTGACTTTACAGAAGGAGATTA-3'; PGT forward primer, 5'-ATCCCC AAAGCACCTGGTTT-3'; PGT reverse primer, 5'-AGAGGCCAAGATAGTCCTGGTAA-3'; PGT VIC probe, 5'-CCATCCATGTCCTCATCTC- $3{ }^{1.14}$ The PCR conditions were $95^{\circ} \mathrm{C}$ for 10 minutes followed by 45 cycles of $95^{\circ} \mathrm{C}$ for 15 seconds, and $60^{\circ} \mathrm{C}$ for 1 minute. ${ }^{14}$ To compare the $F$. nucleatum DNA amounts between tumor DNA samples, the relative values $\left(2^{-\mathrm{ACt}}\right)$ calculated from the threshold cycle $(\mathrm{Ct})$ values for $F$. nucleatum normalized to PGT were used. The qPCR method was validated using serially-diluted F. nucleatum genomic DNA samples (25586D-5; 
ATCC, Manassas, VA, USA). The results of the validation analysis are summarized in Supplementary Fig. S1. F. nucleatumpositive CRCs were further classified into two subgroups (F. nucleatum-high or $F$. nucleatum-low) using a cut-off median value of $2^{-\Delta C t}$. Among the samples of the initial 747 cases subjected to F. nucleatum qPCR analysis, those of 154 cases were determined as failed or inadequate, based on non-evaluable or high $\mathrm{Ct}$ values of PGT. Thus, 593 cases were finally included in this study. The qPCR experiment of each sample was performed independently in triplicate.

\section{DNA analyses for MSI, CIMP, KRAS, and BRAF}

Major molecular factors, including MSI, CIMP, and KRAS/ $B R A F$ mutations, in the CRC samples were analyzed as previously described. ${ }^{18}$ Genomic DNA of each tumor was isolated from representative FFPE tissue blocks by microdissection. MSI testing was performed by DNA fragment analysis using five microsatellite markers (BAT-25, BAT-26, D5S346, D17S250, and D2S123) according to the Bethesda guideline. ${ }^{19}$ MSI status of each case was classified into one of the three categories: MSI-high, MSI-low, and microsatellite stable (MSS). CIMP analysis was carried out by the real-time PCR-based MethyLight assay using eight CIMP markers (MLH1, NEUROG1, CRABP1, CACNA1G, CDKN2A, IGF2, SOCS1, and RUNX3) as previously described. ${ }^{18}$ CIMP status of each case was classified into one of the three cat- egories: CIMP-high, CIMP-low, and CIMP-negative. Mutational status of KRAS exon 2 codons 12 and 13 and BRAF exon 15 codon 600 were examined by Sanger sequencing.

\section{Statistical analyses}

All statistical analyses in this study were performed using SPSS ver. 23 (IBM Corp., Armonk, NY, USA). Comparison analysis between categorical variables was conducted using chisquare test or Fisher exact test. Univariate and multivariate survival analyses were carried out using the Kaplan-Meier method with log-rank test and Cox proportional hazards regression model. All p-values were considered to indicate statistically significant differences if less than 0.05 .

\section{RESULTS}

\section{Variable amounts of $F$. nucleatum according to tumor location bowel subsite in CRCs}

Among the 593 stage II/III CRCs treated with oxaliplatinbased adjuvant chemotherapy (FOLFOX or CAPOX), intratumoral $F$. nucleatum DNA was detected in 408 cases (68.8\%). Each F. nucleatum-positive CRC was classified as F. nucleatum-high or -low based on $F$. nucleatum DNA load, using a cut-off median value of $2^{-\mathrm{ACt}}$. The proportions of $F$. nucleatum-high, -low, and -negative CRCs along the tumor location bowel subsite varied

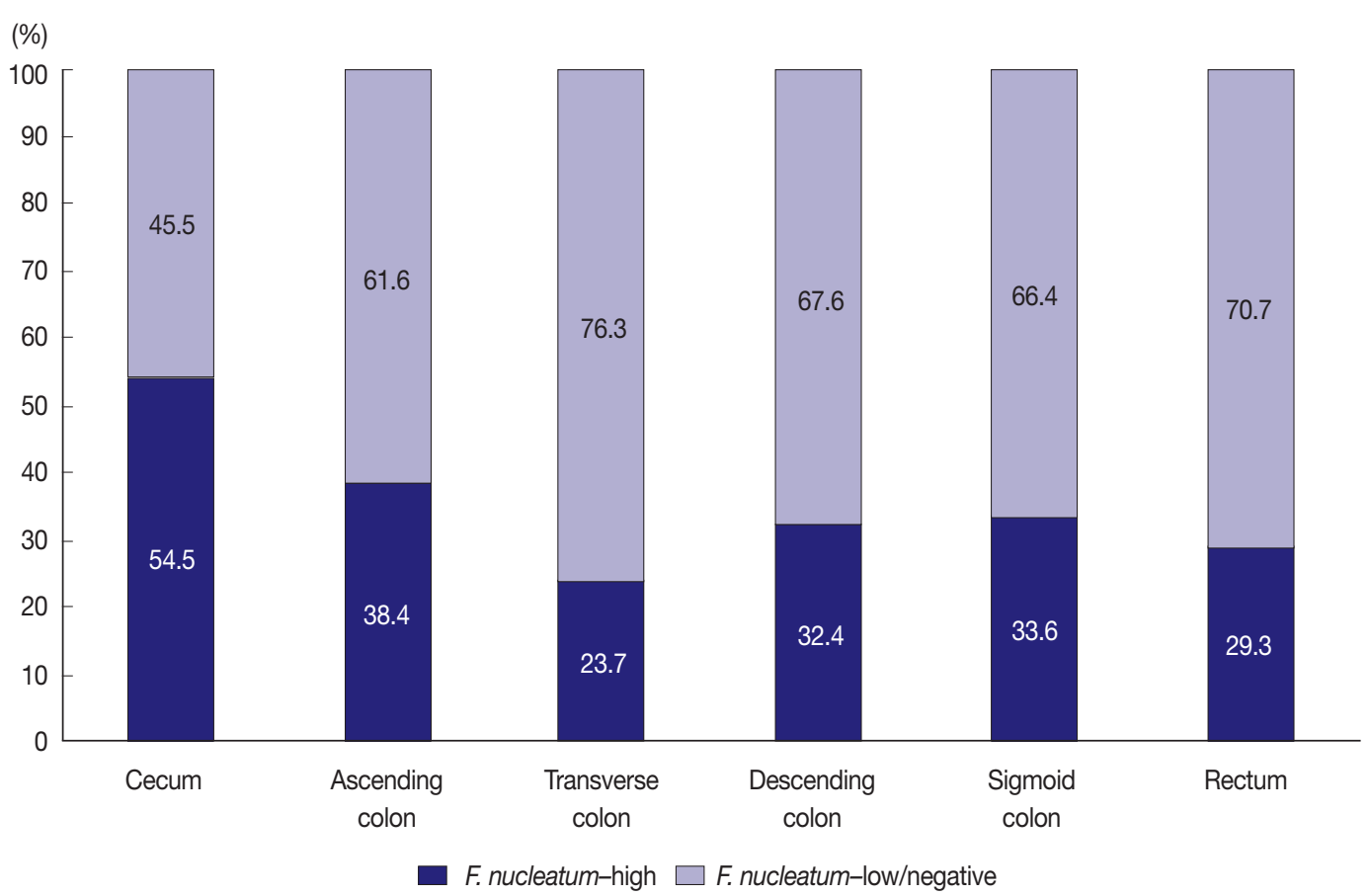

Fig. 1. Different proportions of Fusobacterium nucleatum-high vs F. nucleatum-low/negative colorectal cancers according to tumor location bowel subsites. 
(Fig. 1). The proportion of $F$. nucleatum-high tumors was highest among cecal cancers, whereas that of $F$. nucleatum-high tumors was lowest among transverse colon cancers $(54.5 \%$ and $23.7 \%$, respectively) (Fig. 1).

\section{Clinicopathological and molecular associations of F. nucleatum in CRCs}

We analyzed the relationship between $F$. nucleatum status (high vs. low/negative) and clinicopathological (age, sex, tumor sidedness, $\mathrm{pT} / \mathrm{pN}$ categories, tumor grade, lymphovascular and perineural invasions, and mucinous histology) and molecular characteristics (MSI, CIMP, and KRAS/BRAF mutations) in overall stage II/III CRCs treated with oxaliplatin-based adjuvant chemotherapy $(n=593)$. The results are summarized in Table 1. Among the variables, the $\mathrm{pT}$ category was the only factor with statistical significance. F. nucleatum-high was significantly associated with advanced $\mathrm{pT}$ stage $(\mathrm{pT} 3 / \mathrm{pT} 4)(\mathrm{p}=.005)$ (Table 1$)$. CIMP-high and KRAS mutations were more frequent in $F$. nucleatum-high CRCs than in F. nucleatum-low/negative CRCs, without statistical significance $(\mathrm{p}=.174$ and $\mathrm{p}=.093$, respectively) (Table 1).

\section{Prognostic impact of $F$. nucleatum in CRCs treated with adjuvant chemotherapy}

In survival analysis, no significant difference in disease-free survival (DFS) was evident between the F. nucleatum high and $F$. nucleatum low/negative groups in overall 593 stage II/III CRC patients treated with oxaliplatin-based adjuvant chemotherapy $(\log$-rank $\mathrm{p}=.671)($ Fig. $2 \mathrm{~A})$. In addition, the prognostic significance of $F$. nucleatum was not identified in subgroups stratified by MSI status (log-rank $\mathrm{p}=.858$ in MSI-high CRCs $(\mathrm{n}=40), \log$ rank $\mathrm{p}=.625$ in MSS/MSI-low CRCs ( $\mathrm{n}=545$ ) (Supplementary Fig. S2). However, subgroup analyses according to tumor location demonstrated that DFS of the $F$. nucleatum-high group was significantly better than that of the F. nucleatum-low/negative group in patients with adjuvant FOLFOX or CAPOX-treated colon cancer located in the non-sigmoid colon (from cecum to descending colon, $\mathrm{n}=219)(\log -$ rank $\mathrm{p}=.026)($ Fig. $2 \mathrm{~B})$. In sigmoid colon and rectal cancer patients treated with oxaliplatinbased adjuvant chemotherapy $(\mathrm{n}=374)$, the $F$. nucleatum-high group showed a tendency toward worse DFS compared to the $F$. nucleatum-low/negative group, but this survival difference was not statistically significant (log-rank $\mathrm{p}=.199)$ (Fig. 2C). In multivariate analysis, F. nucleatum-high was an independently favorable prognostic factor in non-sigmoid colon cancer patients treated with oxaliplatin-based adjuvant chemotherapy (hazard ratio, $0.42 ; 95 \%$ confidence interval, 0.18 to $0.97 ; p=.043$ ) (Table 2).
Table 1. Characteristics of adjuvant chemotherapy-treated, stage II/III CRCs according to the Fusobacterium nucleatum status

\begin{tabular}{|c|c|c|c|}
\hline Variable & F. nucleatum-high & $\begin{array}{c}\text { F. nucleatum-low/ } \\
\text { negative }\end{array}$ & p-value \\
\hline Age & & & .286 \\
\hline Younger (<59 yr) & $84(41.2)$ & $178(45.8)$ & \\
\hline Older ( $\geq 59 \mathrm{yr})$ & $120(58.8)$ & $211(54.2)$ & \\
\hline Sex & & & .925 \\
\hline Male & $124(60.8)$ & 238 (61.2) & \\
\hline Female & 80 (39.2) & $151(38.8)$ & \\
\hline Tumor sidedness & & & .287 \\
\hline Right-sided & $69(33.8)$ & 115 (29.6) & \\
\hline Left-sided & $135(66.2)$ & $274(70.4)$ & \\
\hline Gross tumor type & & & .243 \\
\hline Polypoid/fungating & 119 (58.3) & 246 (63.2) & \\
\hline Ulceroinfiltrative & $85(41.7)$ & $143(36.8)$ & \\
\hline pT category & & & .005 \\
\hline pT1/ pT2 & $9(4.4)$ & 44 (11.3) & \\
\hline pT3/pT4 & 195 (95.6) & $345(88.7)$ & \\
\hline pN category & & & .464 \\
\hline pNO & $34(16.7)$ & $56(14.4)$ & \\
\hline $\mathrm{pN1/pN2}$ & $170(83.3)$ & $333(85.6)$ & \\
\hline Tumor histological grade & & & .687 \\
\hline G1/G2 & $188(92.2)$ & $362(93.1)$ & \\
\hline G3/G4 & $16(7.8)$ & $27(6.9)$ & \\
\hline Lymphovascular invasion & & & .419 \\
\hline Absent & $112(54.9)$ & $200(51.4)$ & \\
\hline Present & $92(45.1)$ & $189(48.6)$ & \\
\hline Perineural invasion & & & .171 \\
\hline Absent & $143(70.1)$ & $293(75.3)$ & \\
\hline Present & $61(29.9)$ & $96(24.7)$ & \\
\hline Mucinous histology & & & .269 \\
\hline Absent & $184(90.2)$ & $361(92.8)$ & \\
\hline Present & $20(9.8)$ & $28(7.2)$ & \\
\hline MSI status ${ }^{a}$ & & & .647 \\
\hline MSS/MSI-low & $185(92.5)$ & $360(93.5)$ & \\
\hline MSI-high & $15(7.5)$ & $25(6.5)$ & \\
\hline CIMP status $^{b}$ & & & .174 \\
\hline CIMP-low/negative & 189 (92.6) & 369 (95.3) & \\
\hline CIMP-high & $15(7.4)$ & $18(4.7)$ & \\
\hline KRAS mutation ${ }^{c}$ & & & .093 \\
\hline Absent & 137 (67.2) & $286(73.7)$ & \\
\hline Present & 67 (32.8) & $102(26.3)$ & \\
\hline BRAF mutation & & & .213 \\
\hline Absent & $200(98)$ & 374 (96.1) & \\
\hline Present & 4 (2) & 15 (3.9) & \\
\hline
\end{tabular}

Values are presented as number (\%).

CRC, colorectal cancer; G1, grade 1 (well differentiated); G2, grade 2 (moderately differentiated); G3, grade 3 (poorly differentiated); G4, grade 4 (undifferentiated); MSI, microsatellite instability; MSS, microsatellite-stable; $\mathrm{CIMP}, \mathrm{CpG}$ island methylator phenotype.

${ }^{a}$ Among the 593 cases, MSI status could not be determined in eight cases due to inadequate DNA quality or quantity; ${ }^{b}$ Among the 593 cases, CIMP status could not be determined in two cases due to inadequate DNA quali-

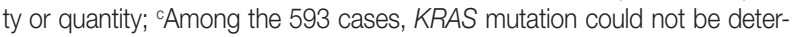
mined in one case due to inadequate DNA quality or quantity. 
All stage II/III colorectal cancers treated with adjuvant chemotherapy $(n=593)$

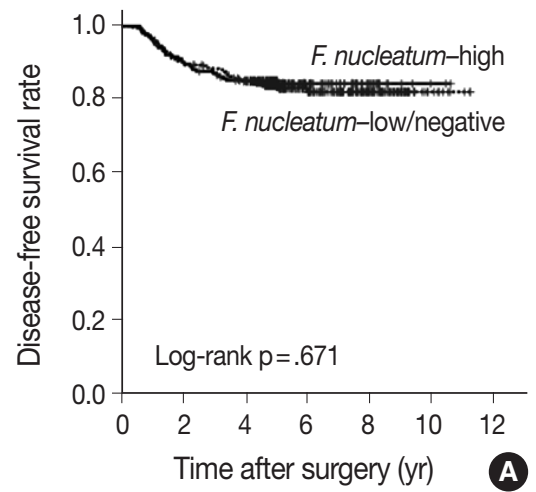

Non-sigmoid colon cancers treated with adjuvant chemotherapy $(n=219)$

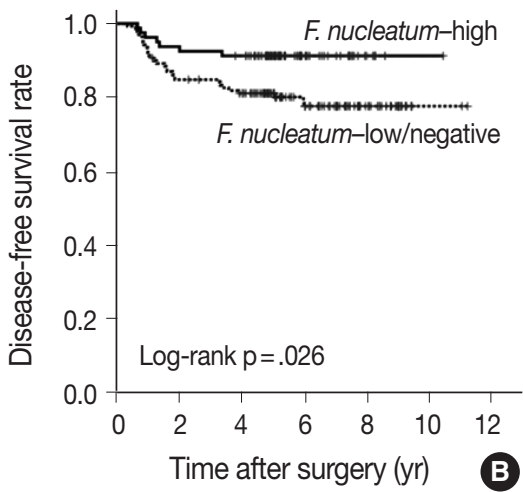

Sigmoid colon or rectal cancers treated with adjuvant chemotherapy $(n=374)$

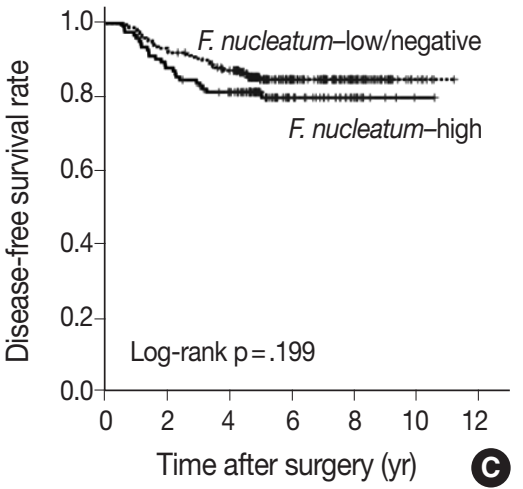

Fig. 2 . Kaplan-Meier survival analysis, including subgroup analysis according to tumor location. (A) No significant difference in disease-free survival was evident between Fusobacterium nucleatum-high and -low/negative subgroups in the overall 593 stage I//II colorectal cancer patients treated with oxaliplatin-based adjuvant chemotherapy. (B) The F. nucleatum-high subgroup was significantly associated with better disease-free survival in non-sigmoid colon cancer patients treated with oxaliplatin-based adjuvant chemotherapy $(n=219)$. (C) In sigmoid coIon and rectal cancer patients treated with oxaliplatin-based adjuvant chemotherapy $(n=374)$, the $F$. nucleatum-high subgroup shows a tendency toward worse disease-free survival without statistical significance.

To further identify the molecular basis of the favorable prognostic effect of $F$. nucleatum observed in non-sigmoid colon cancers, we analyzed the prognostic impact of $F$. nucleatum in subsets of nonsigmoid colon cancer patients according to MSI status. In an MSS/ MSI-low subset of non-sigmoid colon cancer patients treated with adjuvant chemotherapy $(\mathrm{n}=185)$, DFS was significantly better in the $F$. nucleatum-high group than in the $F$. nucleatum-low/negative group (log-rank $\mathrm{p}=.014)$ (Fig. 3A). However, significant DFS difference according to $F$. nucleatum status was not observed in an MSI-high subset of non-sigmoid colon cancer patients ( $\mathrm{n}=$ 31) $(\log$-rank $\mathrm{p}=.844)$ (Fig. 3B). Finally, survival analyses in MSS/MSI-low ( $\mathrm{n}=360)$ and MSI-high $(\mathrm{n}=9)$ subgroups of sigmoid colon or rectal cancers treated with oxaliplatin-based adjuvant chemotherapy demonstrated tendencies toward worse DFS of $F$. nucleatum-high group than of $F$. nucleatum-low/negative group, but there was no statistical significance $(\log$-rank $\mathrm{p}=.193$ in MSS/MSI-low subgroup, Fig. 3C; log-rank $\mathrm{p}=.885$ in MSIhigh subgroup, Fig. 3D)

\section{DISCUSSION}

Direct or indirect roles of gut microbiota in the pathogenesis of a variety of human diseases have been recently proposed. The demonstration of the close association between $F$. nucleatum and CRC has prompted exploration of the pathogenetic, prognostic, and predictive roles of $F$. nucleatum in CRC. However, there are still limited data regarding the prognostic and predictive values of $F$. nucleatum in CRC. Several studies using clinical samples have indicated that intratumoral $F$. nucleatum is potentially associated with poor prognosis in CRC patients. ${ }^{3,11,20}$ Moreover, an experimental study suggested that $F$. nucleatum might be able to induce resistance to chemotherapy by modulating autophagy in CRC cells. ${ }^{4}$ Based on the emerging prognostic significance and potential predictive value of $F$. nucleatum in CRC, we decided to investigate the prognostic relevance of $F$. nucleatum in CRCs treated with adjuvant chemotherapy. Most patients with stage III or high-risk stage II CRC are treated with adjuvant chemotherapy after curative surgery to prevent tumor recurrence. Thus, we collected a large series of stage III or high-risk stage II CRCs treated with oxaliplatin-based adjuvant chemotherapy. The survival differences in patient subgroups according to DNA amount of intratumoral $F$. nucleatum measured by qPCR were statistically analyzed. We found that a high load of intratumoral $F$. nucleatum was independently correlated with improved survival in patients with stage II/III non-sigmoid colon cancer treated with oxaliplatinbased adjuvant chemotherapy (Table 2).

There is a discrepancy between our research and previous studies. Several previous studies revealed that $F$. nucleatum-high CRC patients group tended to have shorter disease-specific survival than $F$. nucleatum-low/negative CRC patients group., ${ }^{3,11,20}$ However, in the current study, F. nucleatum had different prognostic impacts based on tumor location in CRCs treated with adjuvant chemotherapy. In detail, tumors with high levels of $F$. nucleatum had better prognosis than those with low or negative levels of $F$. nucleatum in non-sigmoid colon cancers, including cecum, ascending colon, transverse colon, and descending colon 
Table 2. Univariate and multivariate survival analyses of patients with stage I//III non-sigmoid colon cancer treated with oxaliplatin-based adjuvant chemotherapy $(n=219)$

\begin{tabular}{|c|c|c|c|c|c|}
\hline Variable & No. & $\begin{array}{l}\text { Univariate analysis } \\
\text { HR (95\% Cl) }\end{array}$ & $p$-value & $\begin{array}{c}\text { Multivariate analysis } \\
\text { HR }(95 \% \mathrm{Cl})\end{array}$ & $p$-value \\
\hline \multicolumn{6}{|l|}{ F. nucleatum } \\
\hline F. nucleatum-low/negative & 139 & Reference & & Reference & \\
\hline F. nucleatum-high & 80 & $0.4(0.18-0.92)$ & .031 & $0.42(0.18-0.97)$ & .043 \\
\hline \multicolumn{6}{|l|}{ pT category } \\
\hline pT1/pT2/pT3 & 183 & Reference & & Reference & \\
\hline pT4 & 36 & $5.13(2.65-9.92)$ & $<.001$ & $5.04(2.53-10.07)$ & $<.001$ \\
\hline \multicolumn{6}{|l|}{$\mathrm{pN}$ category } \\
\hline pNo/pN1 & 176 & Reference & & Reference & \\
\hline pN2 & 43 & $2.88(1.47-5.64)$ & .002 & $2.65(1.31-5.35)$ & .007 \\
\hline \multicolumn{6}{|l|}{ Lymphovascular invasion } \\
\hline Absent & 129 & Reference & & Reference & \\
\hline Present & 90 & $2.78(1.41-5.50)$ & .003 & $1.39(0.66-2.95)$ & .387 \\
\hline \multicolumn{6}{|l|}{ Perineural invasion } \\
\hline Absent & 169 & Reference & & Reference & \\
\hline Present & 50 & $2.81(1.45-5.45)$ & .002 & $2.92(1.41-6.05)$ & .004 \\
\hline \multicolumn{6}{|l|}{ BRAF mutation } \\
\hline Absent & 204 & Reference & & Reference & \\
\hline Present & 15 & $3.12(1.30-7.49)$ & .011 & $2.21(0.86-5.69)$ & .1 \\
\hline \multicolumn{6}{|l|}{ Tumor histological grade } \\
\hline G1/G2 & 190 & Reference & & - & \\
\hline G3/G4 & 29 & $1.14(0.44-2.92)$ & .791 & - & - \\
\hline \multicolumn{6}{|l|}{ MSI status ${ }^{a}$} \\
\hline MSS/MSI-low & 185 & Reference & & - & \\
\hline MSI-high & 31 & $0.57(0.17-1.87)$ & .353 & - & - \\
\hline \multicolumn{6}{|l|}{ CIMP status $^{b}$} \\
\hline CIMP-low/negative & 192 & Reference & & - & \\
\hline CIMP-high & 25 & $1.32(0.51-3.40)$ & .567 & - & - \\
\hline \multicolumn{6}{|l|}{ KRAS mutation ${ }^{c}$} \\
\hline Absent & 148 & Reference & & - & \\
\hline Present & 71 & $0.93(0.46-1.89)$ & .844 & - & - \\
\hline
\end{tabular}

HR, hazard ratio; 95\% Cl, 95\% confidence interval of HR; G1, grade 1 (well differentiated); G2, grade 2 (moderately differentiated); G3, grade 3 (poorly differentiated); G4, grade 4 (undifferentiated); MSI, microsatellite instability; MSS, microsatellite-stable; CIMP, CpG island methylator phenotype.

${ }^{a}$ Among the 219 cases, MSI status could not be determined in three cases due to inadequate DNA quality or quantity; 'Among the 219 cases, CIMP status could not be determined in two cases due to inadequate DNA quality or quantity.

cancers (Table 2, Fig. 2B). On the other hand, F. nucleatum-high CRCs showed a tendency toward worse prognosis compared to F. nucleatum-low/negative CRCs in sigmoid colon and rectal cancers (Fig. 2C). Since these contrasting prognostic implications of $F$. nucleatum according to tumor location may counterbalance the overall prognostic effect of $F$. nucleatum in CRCs, presently $F$. nucleatum displayed no association with prognosis in a total of 593 stage II/III CRC patients treated with adjuvant chemotherapy (Fig. 2A). The reason for the discrepancy between the current and prior findings may be the difference in the composition of the study populations. Yamaoka et al. ${ }^{20}$ described that $F$. nucleatum was highly correlated with shorter disease specific survival especially in stage IV CRCs. In that study, in all stages of CRCs, diseasespecific survival was decreased in CRCs featuring a high level of
F. nucleatum compared with that in CRCs with low levels of $F$. nucleatum, although the survival differences according to $F . n u$ cleatum level was decreased compared to that in the stage IV CRC subgroup. ${ }^{20}$ In addition, it cannot be excluded that there might be heterogeneities of detailed treatment approaches, such as adjuvant chemotherapy regimen, in the CRC cohorts of other studies. By contrast, our study samples were a well-selected and relativelyhomogeneous cohort that contained only stage III or high-risk stage II CRCs treated with oxaliplatin-based adjuvant chemotherapy. Therefore, the prognostic implications of $F$. nucleatum in CRC that is evident from our study could be meaningfully different from the results of other research groups.

In an experimental study, F. nucleatum promoted resistance to chemotherapy in CRC cells. ${ }^{4}$ However, our results indicate that 

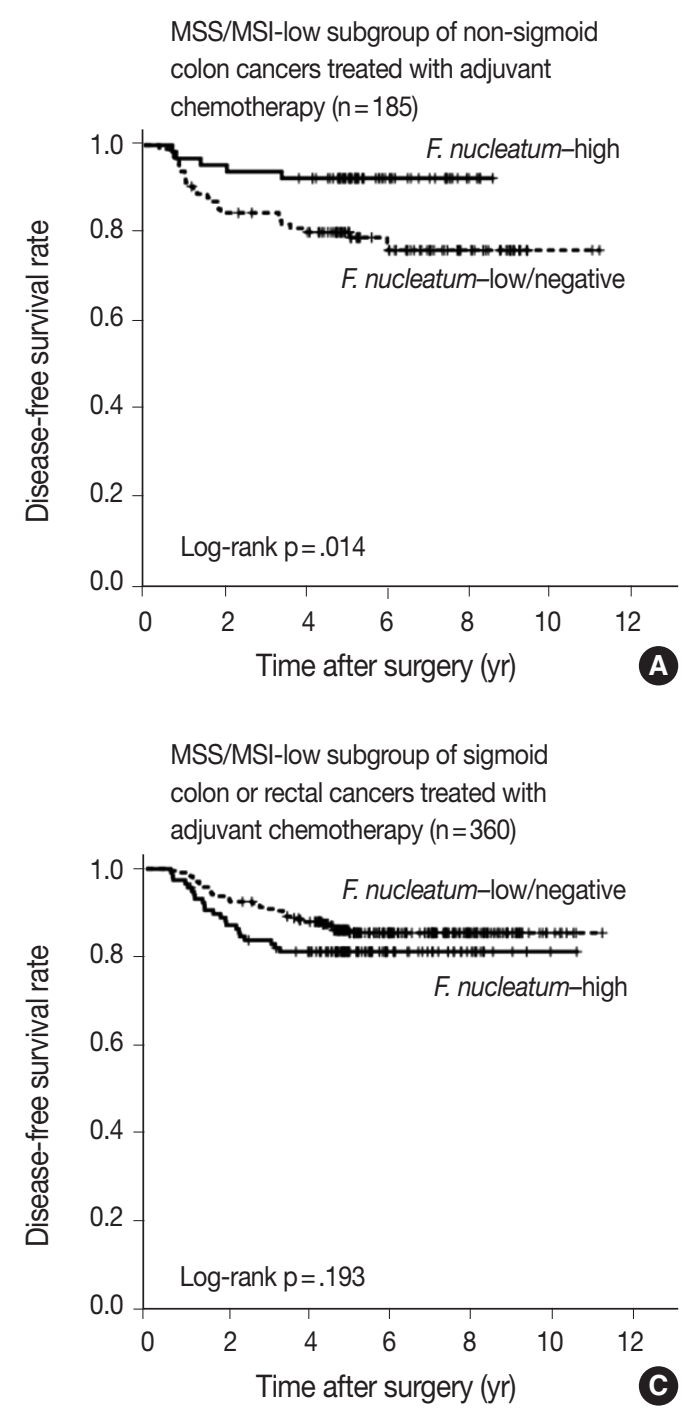
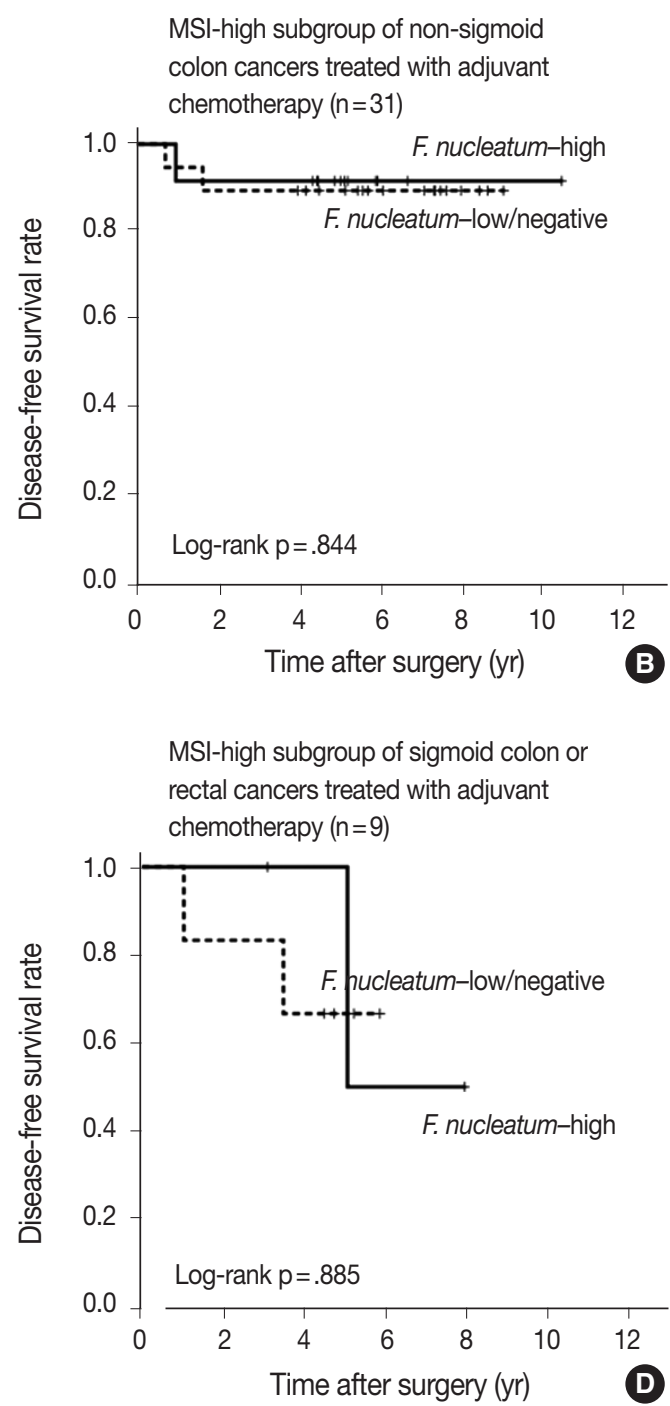

Fig. 3. Kaplan-Meier survival analysis, including subgroup analysis according to combined tumor location and microsatellite instability (MSI) status. (A) The Fusobacterium nucleatum-high subgroup was significantly associated with better disease-free survival in an MSS/MSI-low subset of non-sigmoid colon cancer patients treated with oxaliplatin-based adjuvant chemotherapy $(n=185)$. (B) No significant survival difference according to $F$. nucleatum status was observed in an MSI-high subset of non-sigmoid colon cancer patients treated with oxaliplatinbased adjuvant chemotherapy $(n=31)$. (C) There is a tendency toward worse survival in the $F$. nucleatum-high subgroup without statistical significance in an MSS/MSI-low subset of sigmoid colon or rectal cancer patients treated with oxaliplatin-based adjuvant chemotherapy $(n=$ 360). (D) No significant survival difference according to F. nucleatum status was observed in an MSI-high subset of sigmoid colon or rectal cancer patients treated with oxaliplatin-based adjuvant chemotherapy $(n=9)$.

influences of $F$. nucleatum on responses to chemotherapy might be diverse in the context of tumor location of CRCs. In sigmoid colon and rectal cancers, the expected chemoresistant effect of $F$. nucleatum seems to have occurred because $F$. nucleatum-high was linked with poor prognosis in sigmoid colon and rectal cancer patients treated with adjuvant chemotherapy, although statistical significance was not reached (Fig. 2C). Nevertheless, in nonsigmoid colon cancers, a chemoresistant role of $F$. nucleatum seems to be attenuated. Rather, F. nucleatum might induce a chemoresponsive effect because $F$. nucleatum-high was significantly asso- ciated with favorable prognosis in non-sigmoid colon cancers treated with adjuvant chemotherapy (Table 2, Fig. 2B). The underlying mechanism of the potential contrasting effects of $F$. nucleatum on the chemotherapy response depending on location of CRC is unclear. However, the idea that different tumor locations can define different prognosis and treatment responses in CRC has been increasingly addressed. In fact, based on the accumulating clinical data, primary tumor location is regarded as a prognostic factor in metastatic CRCs. ${ }^{21}$ Stage IV CRCs primarily located in the right-sided colon are significantly associated with worse prognosis 
compared with left-sided stage IV CRCs. The different molecular, pathological, and clinical features between right-sided colon cancers and left-sided CRCs have been reported. ${ }^{21,22}$ Therefore, the potential different impacts of $F$. nucleatum on prognosis and treatment responses according to tumor location in CRCs are not surprising. To the best of our knowledge, this study is the first report to investigate the prognostic effect of $F$. nucleatum according to tumor location in CRCs, especially in adjuvant chemotherapy-treated CRCs. Our study suggests that the prognostic effect of $F$. nucleatum should be evaluated considering the location of the tumor.

In this study, the proportion of F. nucleatum-high CRCs differed in each tumor location bowel subsite. The proportion of $F$. nucleatum-high tumors in all the CRCs was $34.4 \%$ (204 of 593). Cecal cancers displayed the highest proportion of $F$. nucleatumhigh tumors $(54.5 \%)$, followed by ascending colon cancers $(38.4 \%)$ (Fig. 1). It was notable that over half of the cecal cancers were $F$. nucleatum-high tumors. Our results are consistent with those of previous studies demonstrating the significant association of the proximal location of CRCs with a high level of intratumoral $F$. nucleatum. According to the study by Mima et al., ${ }^{13}$ the proportion of F. nucleatum-high CRCs increased along the distance from the anal verge, and cecal cancers showed the highest proportion of $F$. nucleatum-high subtype. The underlying mechanism of the specific enrichment of $F$. nucleatum in cecal and ascending colon cancers is still unclear, but microenvironmental or biological factors specifically found in the cecal to ascending colon areas could influence the increase of intratumoral $F$. nucleatum. For example, bacterial biofilms are intensively enriched in rightsided colon tumors compared with those in left-sided colorectal tumors. ${ }^{23}$ Based on recent experimental findings, potential molecular mechanisms can be hypothesized. According to a previous experimental study, F. nucleatum is enriched in colorectal tumor tissue by Fap2 binding to Gal-GalNAc expressed on tumor cells. $^{24}$ Thus, it can be hypothesized that Gal-GalNAc expression on tumor cells might be more upregulated in the rightsided colon than in the left-sided colon. Further investigations are needed to elucidate the biological reason of the preference of invasive $F$. nucleatum for right-sided colon cancers.

According to the recent data reported by Ogino group, F. nucleatum in CRCs differentially impacts tumor-infiltrating lymphocyte (TIL) density depending on MSI status. ${ }^{25}$ In detail, there was an inverse association between $F$. nucleatum load and TIL density in MSI-high CRCs, whereas a positive correlation between $F$. nucleatum load and TIL density was observed in nonMSI-high CRCs. ${ }^{25}$ This finding can provide an important clue for the interpretation of our present results. It has been validated that high TIL density is strongly associated with favorable prognosis in CRCs. ${ }^{26}$ Thus, because F. nucleatum-high tumors might be associated with increased antitumor immunity and subsequent improved prognosis in non-MSI-high CRCs, the favorable prognostic effect of $F$. nucleatum-high in the MSS/MSI-low subset of non-sigmoid colon cancers, which was observed in our present study, could be a reasonable finding. However, we also found that the prognostic significance of $F$. nucleatum was valid only in non-sigmoid colon cancers, but not in sigmoid colon/ rectal cancers, suggesting that both tumor location and MSI status should be concurrently considered for understanding the prognostic implications of $F$. nucleatum in CRCs.

There have been several reports regarding the poor prognostic effect of $F$. nucleatum in CRCs, which was mainly observed in Western CRC cohorts or stage IV CRC cohorts. ${ }^{3,20,27}$ However, our present data indicate that high intratumoral $F$. nucleatum load might be associated with favorable prognosis in a limited subgroup of CRCs, a MSS/MSI-low subset of non-sigmoid colon cancers. We suspect that different compositions of tumor locations and MSI subtypes in CRC cohorts might influence the different prognostic effects of $F$. nucleatum in overall CRCs. Because it has been known that the frequency of MSI-high in CRCs is definitely lower in East Asia countries than in Western countries, ${ }^{28}$ the potential favorable prognostic effect of $F$. nucleatum in proximal colonic-located, non-MSI-high CRCs might be significantly attenuated in CRC cohorts of Western countries, which consist of relatively high numbers of MSI-high tumors. Instead, both the tendency toward worse prognosis of $F$. nucleatum-high in MSI-high tumors (Supplementary Fig. S2A) and the potential poor prognostic effect of $F$. nucleatum-high tumors observed in sigmoid colon/rectal cancers (Fig. 2C) might augment the adverse prognostic impact of $F$. nucleatum in overall CRCs. To confirm this hypothesis, additional investigations using various CRC cohorts having different ethnic backgrounds would be needed. Regarding the poor prognostic feature of $F$. nucleatum in stage IV CRCs observed in a few studies, ${ }^{20,27}$ it could be explained by relatively high proportion of distal-located CRCs as primary origin of stage IV CRCs. Thus, the potential worse prognostic effect of $F$. nucleatum in sigmoid colon or rectal cancers might be augmented especially in a stage IV subset of CRCs.

Although significant associations between CIMP-high (and/ or MSI-high) and F. nucleatum in CRCs were reported in several previous studies, ${ }^{3,8,9}$ significant correlation between F. nucleatum-high group and CIMP-high or MSI-high molecular subtype was not observed in our present study (Table 1). However, there was an evident tendency toward higher proportion of 
CIMP-high tumors in F. nucleatum-high group than in F. nucleatum-low/negative group (7.4\% vs. $4.7 \%$ ) (Table 1$)$. In addition, we performed mean comparison of $F$. nucleatum DNA amount $\left(2^{-\Delta C t}\right)$ between CIMP-high and CIMP-low/negative tumors, and the results indicated that mean $F$. nucleatum DNA amount was higher in CIMP-high tumors than in CIMP-low/negative tumors although statistical significance was not reached (0.986 vs. 0.367, p = .157) (Supplementary Fig. S3). The reason for unclear molecular association of $F$. nucleatum in our study samples may be explained by potential ethnic differences and biased sample composition. As mentioned above, the frequencies of MSI-high and CIMP-high in CRCs are lower in East Asian population than in Western population. If a high number of CIMPhigh cases were included in our cohort, significant association between $F$. nucleatum-high and CIMP-high might have been observed. Moreover, our study samples were confined to selected stage III or high-risk stage II CRCs treated with adjuvant chemotherapy. Thus, molecular compositions of our CRC cohort were possibly biased. For example, the CIMP-high/non-MSIhigh subtype has been known as an aggressive phenotype of CRCs and can be more enriched in stage IV tumors. Because stage IV cases were excluded from our study samples, the potential association between F. nucleatum-high and CIMP-high could be weakened. Considering that data are limited, the relationship between F. nucleatum and specific molecular phenotypes in CRCs has not been conclusive yet. Therefore, further clinical and experimental investigations are needed to elucidate whether CIMPhigh and/or MSI-high molecular phenotype can significantly interact with intratumoral $F$. nucleatum enrichment in CRCs.

The proportion of F. nucleatum-positive cases in CRCs by qPCR analysis has been variable according to different investigations $(8.6 \%-74 \%) .{ }^{29}$ In our results, F. nucleatum DNA was detected in 408 out of 593 cases $(68.8 \%)$. The reason for variability in the F. nucleatum-positive rate in CRCs is unclear, but tissue quality might be a critical factor for this discrepancy. Recently, Lee et $a .^{27}$ found that the tissue fixation method could affect different results of $F$. nucleatum qPCR analysis. We also found that when the FFPE tissues were more recent, the positive rate of F. nucleatum was increased (unpublished data). Therefore, it can be inferred that $F$. nucleatum-positive rate by qPCR method could be variable, depending on tissue fixation method and tissue storage time.

There are several limitations in this study. First, we assessed the amount of $F$. nucleatum in genomic DNA samples extracted from FFPE tissues. The precise quantification of $F$. nucleatum could be disturbed owing to the degraded nature of DNA extract- ed from FFPE tissues although a substantial number of previous studies that analyzed F. nucleatum in clinical CRC samples also used FFPE tissue-derived DNA. Second, our study cohort was retrospectively collected. The results from our study should be validated by other prospective studies.

In conclusion, the prognostic impact of $F$. nucleatum in CRCs treated with adjuvant chemotherapy may differ depending on the combined status of primary tumor location and MSI molecular phenotype. Intratumoral $F$. nucleatum load may be a potential prognostic factor in stage III or high-risk stage II non-sigmoid colon cancers treated with oxaliplatin-based adjuvant chemotherapy, especially in an MSS/MSI-low molecular subtype. There have been very limited data regarding the detailed prognostic implications of $F$. nucleatum in CRCs according to various clinicopathologic and molecular contexts. Therefore, further studies using large prospective cohorts will be necessary to validate the different location/MSI-dependent prognostic impacts of $F$. nucleatum in CRCs treated with adjuvant chemotherapy.

\section{ORCID}

Hyeon Jeong Oh: https:/orcid.org/0000-0002-9998-3988

Jung Ho Kim: https://orcid.org/0000-0002-6031-3629

Jeong Mo Bae:: https:/orcid.org/0000-0003-0462-3072

Hyun Jung Kim: https:/orcid.org/0000-0002-8136-7095

Nam-Yun Cho: https://orcid.org/0000-0002-2126-9549

Gyeong Hoon Kang: https://orcid.org/0000-0003-2380-6675

\section{Electronic Supplementary Material}

Supplementary materials are available at Journal of Pathology and Translational Medicine (http://jpatholtm.org).

\section{Conflicts of Interest}

The authors declare that they have no potential conflicts of interest.

\section{Acknowledgments}

This study was supported by the National Research Foundation of Korea grant funded by the Korea government (Ministry of Science and ICT) (2016R1C1B2010627), a grant from the National Research Foundation of Korea grants funded by the Korea government (Ministry of Science and ICT) (2016M3A9B 6026921), and a grant from the Korea Health Technology R\&D Project through the Korea Health Industry Development Institute funded by the Korea government (Ministry of Health and Welfare) (HI14C1277). 


\section{REFERENCES}

1. Kostic AD, Chun E, Robertson L, et al. Fusobacterium nucleatum potentiates intestinal tumorigenesis and modulates the tumorimmune microenvironment. Cell Host Microbe 2013; 14: 207-15.

2. Rubinstein MR, Wang X, Liu W, Hao Y, Cai G, Han YW. Fusobacterium nucleatum promotes colorectal carcinogenesis by modulating E-cadherin/beta-catenin signaling via its FadA adhesin. Cell Host Microbe 2013; 14: 195-206.

3. Mima K, Nishihara R, Qian ZR, et al. Fusobacterium nucleatum in colorectal carcinoma tissue and patient prognosis. Gut 2016; 65: 1973-80.

4. Yu T, Guo F, Yu Y, et al. Fusobacterium nucleatum promotes chemoresistance to colorectal cancer by modulating autophagy. Cell 2017; 170: 548-63.e16.

5. Geller LT, Barzily-Rokni M, Danino T, et al. Potential role of intratumor bacteria in mediating tumor resistance to the chemotherapeutic drug gemcitabine. Science 2017; 357: 1156-60.

6. Kostic AD, Gevers D, Pedamallu CS, et al. Genomic analysis identifies association of Fusobacterium with colorectal carcinoma. Genome Res 2012; 22: 292-8.

7. Castellarin M, Warren RL, Freeman JD, et al. Fusobacterium nucleatum infection is prevalent in human colorectal carcinoma. Genome Res 2012; 22: 299-306.

8. Tahara T, Yamamoto E, Suzuki H, et al. Fusobacterium in colonic flora and molecular features of colorectal carcinoma. Cancer Res 2014; 74: 1311-8.

9. Ito M, Kanno S, Nosho K, et al. Association of Fusobacterium nucleatum with clinical and molecular features in colorectal serrated pathway. Int J Cancer 2015; 137: 1258-68.

10. Yu J, Chen $\mathrm{Y}, \mathrm{Fu} X$, et al. Invasive Fusobacterium nucleatum may play a role in the carcinogenesis of proximal colon cancer through the serrated neoplasia pathway. Int J Cancer 2016; 139: 1318-26.

11. Flanagan L, Schmid J, Ebert M, et al. Fusobacterium nucleatum associates with stages of colorectal neoplasia development, colorectal cancer and disease outcome. Eur J Clin Microbiol Infect Dis 2014; 33: 1381-90.

12. Mima K, Sukawa $Y$, Nishihara R, et al. Fusobacterium nucleatum and T cells in colorectal carcinoma. JAMA Oncol 2015; 1: 653-61.

13. Mima K, Cao Y, Chan AT, et al. Fusobacterium nucleatum in colorectal carcinoma tissue according to tumor location. Clin Transl Gastroenterol 2016; 7: e200.

14. Park HE, Kim JH, Cho NY, Lee HS, Kang GH. Intratumoral Fusobacterium nucleatum abundance correlates with macrophage infiltration and CDKN2A methylation in microsatellite-unstable colorectal carcinoma. Virchows Arch 2017; 471: 329-36.

15. Routy B, Le Chatelier E, Derosa L, et al. Gut microbiome influences efficacy of PD-1-based immunotherapy against epithelial tumors. Science 2018; 359: 91-7.

16. Vetizou M, Pitt JM, Daillère R, et al. Anticancer immunotherapy by CTLA-4 blockade relies on the gut microbiota. Science 2015; 350: 1079-84.

17. Gopalakrishnan V, Spencer CN, Nezi L, et al. Gut microbiome modulates response to anti-PD-1 immunotherapy in melanoma patients. Science 2018; 359: 97-103.

18. Bae JM, Kim JH, Oh HJ, et al. Downregulation of acetyl-CoA synthetase 2 is a metabolic hallmark of tumor progression and aggressiveness in colorectal carcinoma. Mod Pathol 2017; 30: 267-77.

19. Boland CR, Thibodeau SN, Hamilton SR, et al. A National Cancer Institute Workshop on Microsatellite Instability for cancer detection and familial predisposition: development of international criteria for the determination of microsatellite instability in colorectal cancer. Cancer Res 1998; 58: 5248-57.

20. Yamaoka Y, Suehiro Y, Hashimoto S, et al. Fusobacterium nucleatum as a prognostic marker of colorectal cancer in a Japanese population. J Gastroenterol 2018; 53: 517-24.

21. Stintzing S, Tejpar S, Gibbs P, Thiebach L, Lenz HJ. Understanding the role of primary tumour localisation in colorectal cancer treatment and outcomes. Eur J Cancer 2017; 84: 69-80.

22. Bae JM, Kim JH, Kang GH. Molecular subtypes of colorectal cancer and their clinicopathologic features, with an emphasis on the serrated neoplasia pathway. Arch Pathol Lab Med 2016; 140: 406-12.

23. Dejea CM, Wick EC, Hechenbleikner EM, et al. Microbiota organization is a distinct feature of proximal colorectal cancers. Proc Natl Acad Sci U S A 2014; 111: 18321-6.

24. Abed J, Emgård JE, Zamir G, et al. Fap2 mediates Fusobacterium nucleatum colorectal adenocarcinoma enrichment by binding to tumorexpressed Gal-GalNAc. Cell Host Microbe 2016; 20: 215-25.

25. Hamada T, Zhang X, Mima K, et al. Fusobacterium nucleatum in colorectal cancer relates to immune response differentially by tumor microsatellite instability status. Cancer Immunol Res 2018; 6: 132736.

26. Pagès F, Mlecnik B, Marliot F, et al. International validation of the consensus Immunoscore for the classification of colon cancer: a prognostic and accuracy study. Lancet 2018; 391: 2128-39.

27. Lee DW, Han SW, Kang JK, et al. Association between Fusobacterium nucleatum, pathway mutation, and patient prognosis in colorectal cancer. Ann Surg Oncol 2018; 25: 3389-95.

28. Kim JH, Kang GH. Molecular and prognostic heterogeneity of microsatellite-unstable colorectal cancer. World J Gastroenterol 2014; 20: $4230-43$.

29. Shang FM, Liu HL. Fusobacterium nucleatum and colorectal cancer: a review. World J Gastrointest Oncol 2018; 10: 71-81. 